\title{
A Comparative Study on Steganography Digital Images: A Case Study of Scalable Vector Graphics (SVG) and Portable Network Graphics (PNG) Images Formats
}

\author{
Abdulgader Almutairi \\ College of Sciences and Arts in ArRass, \\ Qassim University, Kingdom of Saudi Arabia
}

\begin{abstract}
Today image steganography plays a key role for exchanging a secret data through the internet. However, the optimal choice of images formats for processing steganography is still an open issue; therefore, this research comes into a table. This research conducts a comparative study between Scalable Vector Graphics (SVG) image format and Portable Network Graphics (PNG) image format. As results show, SVG image format is more efficient than PNG image format in terms of capacity and scalability before and after processing steganography. As well, SVG image format helps to increase simplicity and performance for processing steganography, since it is an XML text file. Our comparative study provides significant results between SVG and PNG images, which have not been seen in the previous related studies.
\end{abstract}

Keywords-Image steganography; data hiding; raster and vector images; Scalable Vector Graphics (SVG) and Portable Network Graphics (PNG) images format

\section{INTRODUCTION}

Nowadays, steganography is used widely for delivering a secure message among different parties distributed across the world. Steganography is a Greek term, which means a concealed writing. Steganography comprises four main categories: video steganography, audio steganography, image steganography, and text steganography. It has been observed that image steganography is the most popular steganography type due to images frequencies in the internet, and therefore image steganography type is identified for discussion and review in this research [1]-[4].

A digital image is a numeric form of a two dimensional image that is made of image components known as pixels. Usually, pixels are structured in well-arranged array. The columns number of the array defines width of the image, while the rows number of the array defines image height. Broadly, digital images are two types, namely raster digital images and vector digital images [5].

The raster digital images are bitmaps that are defined through a grid of specific pixels, which jointly structure the digital image. It concentrates digital images as a pool of uncountable small squares called pixels. Every single pixel is coded in a particular sort. It can be produced by an illustration program or generated from a scanner. Thus, raster images can encompass millions of different colors, with each one being represented by a single pixel.

The raster digital images are commonly used for non-line digital images, since they normally involve complicated composition, thin chromatic gradations, and indefinite lines and shapes. The most common spread raster digital images include Joint Photographic Experts Group (JPEG), Windows Bitmap (BMP), Graphics Interchange Format (GIF), and Portable Network Graphics (PNG) images formats [5]-[7].

On the contrary, vector digital images are constructed based on mathematical forms that state geometric features like rectangles, curves, lines, circles, and polygons. In addition, these components are loaded with gradients, color, blends, and tints. Besides that, the lines project a stroke merit like various chunkiness and colors for a solid or dashed line. The vector digital images are used to construct more organized digital images, such as fonts, logos, letterhead. The most commonly used vector digital images are Vector Markup Language (VML) and Scalable Vector Graphics (SVG) images formats [8]-[11]. Therefore, the difference between vector images and raster images is that the vector images are object based and the others are pixel based. The rest of the paper is organized as follows: Section II starts with literature review. Section III gives comparative study on steganography digital images between portable network graphics (PNG) and scalable vector graphics (SVG) images formats. Results discussion and analysis is presented in Section IV. And the paper has been summarized in Section V.

\section{LITERATURE REVIEW}

Broadly, digital images are divided into two main types, namely raster digital images and vector digital images. The former are bitmaps that are well-defined through a net of specific pixels in order to form digital images. The most common spread raster digital images are Joint Photographic Experts Group (JPEG), Graphics Interchange Format (GIF), and Portable Network Graphics (PNG) images formats. The latter are constructed based on mathematical forms that state geometric features like rectangles, curves, lines, circles, and polygons. In addition, these components are loaded with gradients, color, blends, and tints. Besides that, the lines project a stroke merit like various chunkiness and colors for a 
solid or dashed line. The most commonly used vector digital images are Vector Markup Language (VML) and Scalable Vector Graphics (SVG) images formats [2], [5]-[8], [10], [11], [15], [16].

Amongst the various raster's digital images type, a Portable Network Graphics (PNG) image format is the most widely spread raster digital image, which can be attributed to its exceptional features, which are absent in the others raster image formats. Hence, PNG image format is nominated in the current research to conduct a comparative study on steganography digital images. PNG image format is designed to be exchanged smoothly through network, and to work well in online applications like web browsers. The PNG image format provides integrity checking and transmission errors detection. PNG image format is a free and open source format, which was an alternative to GIF. PNG image format supports 8-bit, 16-bit, 24-bit, 32-bit, and 48-bit images, while GIF image format supports only 256 colors and a single transparent color. In addition, PNG image format excels JPEG image format, particularly in case of a big sized image, which losses compression, which means when compressed, it does not lose any data. The animated images format of PNG is MNG and APNG images formats. PNG image format is commonly used for graphs, diagrams, and anywhere to display flat colors and lines, not needing scaling up [2], [5]-[8], [10], [11], [15], [16].

The RGB (Red, Green and Blue) color model is an essential tool, to edit an image's pixels in order to hide a secret data into PNG image formats. The RGB color model is a collective color model, which collects red, green and blue colors together in several means to reproduce a various colors. Fig. 1 presents PNG24 image in RGB color model [6], [17].

Amongst the various vector's digital images type, a Scalable Vector Graphics (SVG) image format is the most widely spread vector digital image. Hence, SVG image format is selected in the current research in order to conduct a comparative study on steganography digital images. SVG image format is an open standard and based on Extensible Markup Language (XML), which is defined in XML text file using any text editor. It presents the qualities of being searchable, scriptable, compressible, and also can be zoomed and indexed. It supports animation and scalability, since its data is saved as a geometric description, instead of the description of each single image pixel in a raster digital image. Table I presents basic comparisons between SVG and PNG images format [2], [5]-[8], [10], [11], [15], [16].

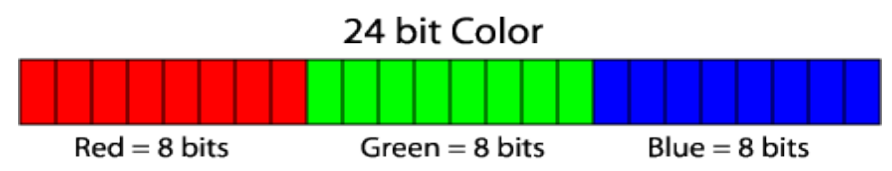

Fig. 1. PNG24 image in RGB color model.

TABLE I. BASIC COMPARISONS BETWEEN SVG AND PNG IMAGE FORMATS.

\begin{tabular}{|l|l|l|l|}
\hline No & Property & $\begin{array}{l}\text { SVG Images } \\
\text { Format }\end{array}$ & $\begin{array}{l}\text { PNG Images } \\
\text { Format }\end{array}$ \\
\hline 1 & Type & Vector Image & Raster Image \\
\hline 2 & Resolution & Unlimited & $\begin{array}{l}\text { Limited to image's } \\
\text { pixels }\end{array}$ \\
\hline 3 & Speed & $\begin{array}{l}\text { Fast, since its file's } \\
\text { size is small. }\end{array}$ & $\begin{array}{l}\text { Slow, since its } \\
\text { file's size often is } \\
\text { larger }\end{array}$ \\
\hline 5 & Animation & $\begin{array}{l}\text { Support animation } \\
\text { (ECMAScript, CSS, } \\
\text { and SMIL) }\end{array}$ & $\begin{array}{l}\text { Support animation } \\
\text { (MNG and APNG } \\
\text { images formats) }\end{array}$ \\
\hline 6 & Zoomable & It can be compressed & $\begin{array}{l}\text { It can be } \\
\text { compressed }\end{array}$ \\
\hline 7 & Open Standard & $\begin{array}{l}\text { Standardized by } \\
\text { W3C }\end{array}$ & $\begin{array}{l}\text { Zooming effects } \\
\text { shadow in the } \\
\text { image. }\end{array}$ \\
\hline
\end{tabular}

III. A COMPARATIVE STUDY ON STEGANOGRAPHY DiGITAL IMAGES BETWEEN PORTABLE NETWORK GRAPHICS (PNG) AND SCALABLE VECTOR GRAPHICS (SVG) IMAGES FORMATS

This section, presents a comparative study on steganography digital images between PNG and SVG images formats. Firstly, it presents SVG and PNG images format sizes prior to steganography processing. This is followed by a discussion on SVG and PNG images format sizes after steganography processing [10]-[15], [18], [19].

\section{A. Comparing SVG and PNG Images Format Sizes before Steganography Processing}

In order to conduct this comparison, we selected the most commonly used three types of image editing software, namely Inkscape image editing software [20], Visio image editing software [21], and Dia image editing software [22]. Afterwards, we used these three image editing software to create and edit the three famous images namely, Lena image, Baboon image, and Pepper image, and to save each image twice; one in SVG image format, and second in PNG image format. After that, we calculated image size for all saved images, and the difference in image size between SVG and PNG image formats. Table II below presents the corresponding findings [5], [6], [7], [17].

TABLE II. A COMPARISON BETWEEN SVG AND PNG IMAGES FormAT SIZES BEFORE STEGANOGRAPHY PROCESSING

\begin{tabular}{|c|l|l|l|l|l|l|l|l|l|}
\hline \multirow{2}{*}{$\begin{array}{c}\text { Image } \\
\text { Name }\end{array}$} & \multicolumn{3}{|c|}{ Inkscape Image Editing Software } & \multicolumn{3}{c|}{ Visio Image Editing Software } & \multicolumn{3}{c|}{ Dia Image Editing Software } \\
\cline { 2 - 10 } & $\begin{array}{l}\text { SVG Size } \\
\text { (Bytes) }\end{array}$ & $\begin{array}{l}\text { PNG Size } \\
\text { (Bytes) }\end{array}$ & $\begin{array}{l}\text { Size Diff. } \\
\text { (Bytes) }\end{array}$ & $\begin{array}{l}\text { SVG Size } \\
\text { (Bytes) }\end{array}$ & $\begin{array}{l}\text { PNG Size } \\
\text { (Bytes) }\end{array}$ & $\begin{array}{l}\text { Size Diff. } \\
\text { (Bytes) }\end{array}$ & $\begin{array}{l}\text { SVG Size } \\
\text { (Bytes) }\end{array}$ & $\begin{array}{l}\text { PNG Size } \\
\text { (Bytes) }\end{array}$ & $\begin{array}{l}\text { Size Diff. } \\
\text { (Bytes) }\end{array}$ \\
\hline Lena & 562,514 & $1,275,975$ & 713,461 & 288,569 & 841,419 & 552,850 & 434 & 336,087 & 335,653 \\
\hline Baboon & 881,448 & $1,161,746$ & 280,298 & $1,083,259$ & $1,322,223$ & 238,964 & 436 & 629,707 & 629,271 \\
\hline Pepper & 18,652 & 645,490 & 626,838 & 187,391 & $1,046,081$ & 858,690 & 437 & 102,015 & 101.578 \\
\hline
\end{tabular}




\section{B. Comparing SVG and PNG Images Format Sizes after Steganography Processing}

In order to perform this comparison, we used OpenStego software, with the purpose to hide a secret data into PNG images format, and notepad text editor software to hide the same secret data into SVG images format [23]. This choice was particularly underpinned by the idea that OpenStego software manipulates images through GRB color model, which is required for processing PNG images format since they are bitmaps images file, and they are only processed through GRB color model. On the other side, notepad text editor software manipulates SVG images format since they are written in XML file format that is a text and not bitmaps, which can be processed through notepad software [1], [24][29]. The secret data is "My Visa Credit Card number is 4539962848122779, CCV number is 483, and Expiration Date is $12 / 17$ ". First, in the study, a secret data was encrypted using AES cryptographic algorithm, which was then hidden (embedded) into SVG and PNG images. As stated earlier, OpenStego software hid the secret data written inside Microsoft word file, into PNG image format, while the notepad software added XML tag into SVG image format. The XML tag merely included an external another XML file, which contained the real encrypted secret data as shown in Fig. 2 below. Hence, SVG image format itself was not be affected when hiding bigger secret data. Besides that, it reflected the simplicity of steganography processing, which is easy for SVG images format compared with PNG images format requiring RGB color model. Moreover, the simplicity of steganography processing usually provides a better performance [1], [16], [24], [30].

Table III below shows the corresponding sizes of SVG and PNG images format after steganography processing.

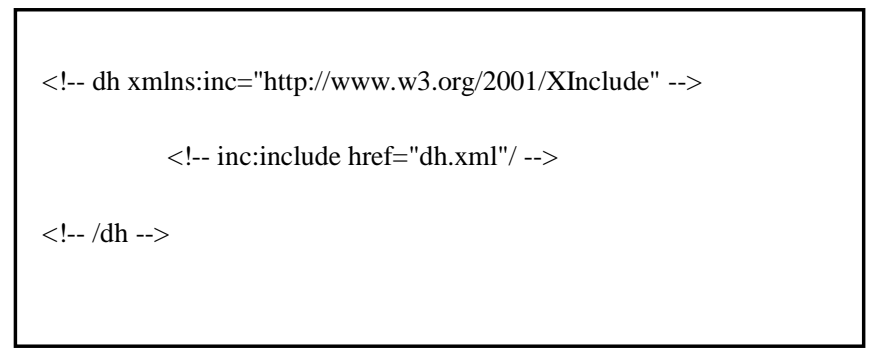

Fig. 2. XML Tag includes an Encrypted Data in SVG Image Format.

\section{RESUlTS DiscusSION AND ANALYSIS}

As shown in Table II, SVG image format occupies less capacity in comparison with PNG image format, which evidences that SVG image format is more efficient than PNG image format in term of image's capacity. For instance, the size of Lena image when saved in SVG image format using Inkscape software is 562,514 Bytes, while its size is $1,275,975$ Bytes when saved in PNG image format. The difference in size of Lena image between SVG and PNG image formats is 713,461 Bytes, which acts $55.9 \%$ of PNG image's size. Likewise, the size of Lena image when saved in SVG image format using Visio software is 288,569 Bytes, while its size is 841,419 Bytes when saved in PNG image format. The difference in size of Lena image between SVG and PNG image formats is thus, 552,850 Bytes, which is $65.7 \%$ of PNG image's size. Similarly, the size of Lena image when saved in SVG image format using Dia software is 434 Bytes, while its size is 336,087 Bytes when saved in PNG image format. The difference in size of Lena image between SVG and PNG image formats is thus, 335,653 Bytes, which is $99.8 \%$ of PNG image's size. Equally, the same calculations are applied to all other images as shown in Table II above. Overall, the difference in size between SVG and PNG image formats before steganography processing is about 18\% - 99.9\%. Correspondingly, Fig. 3, 4, and 5 below show the sizes of SVG and PNG image formats and the difference between them in Inkscape, Visio, and Dia image editing software respectively.

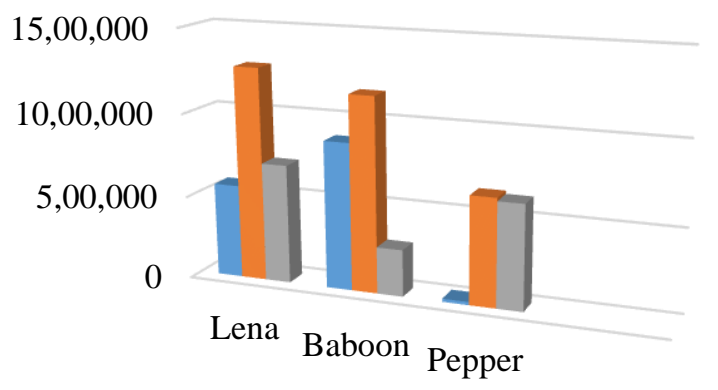

- SVG Size (Bytes) $\square$ PNG Size (Bytes)

Size Diff. (Bytes)

Fig. 3. SVG and PNG image sizes in Inkscape image editing software.

TABLE III. A COMPARISON BETWEEN SVG AND PNG IMAGES FoRMAT SiZES AFTER STEGANOGRAPHy PROCESSING

\begin{tabular}{|l|l|l|l|l|l|l|l|l|l|}
\hline \multirow{2}{*}{$\begin{array}{l}\text { Image } \\
\text { Name }\end{array}$} & \multicolumn{3}{|l|}{ Inkscape Image Editing Software } & \multicolumn{2}{l|}{ Visio Image Editing Software } & \multicolumn{3}{l|}{ Dia Image Editing Software } \\
\cline { 2 - 10 } & $\begin{array}{l}\text { SVG Size } \\
\text { (Bytes) }\end{array}$ & $\begin{array}{l}\text { PNG Size } \\
\text { (Bytes) }\end{array}$ & $\begin{array}{l}\text { Size Diff. } \\
\text { (Bytes) }\end{array}$ & $\begin{array}{l}\text { SVG Size } \\
\text { (Bytes) }\end{array}$ & $\begin{array}{l}\text { PNG Size } \\
\text { (Bytes) }\end{array}$ & $\begin{array}{l}\text { Size Diff. } \\
\text { (Bytes) }\end{array}$ & $\begin{array}{l}\text { SVG Size } \\
\text { (Bytes) }\end{array}$ & $\begin{array}{l}\text { PNG Size } \\
\text { (Bytes) }\end{array}$ & $\begin{array}{l}\text { Size Diff. } \\
\text { (Bytes) }\end{array}$ \\
\hline Lena & 562,628 & $1,825,337$ & $1,262,709$ & 288,681 & 845,107 & 556,426 & 554 & 343,282 & 342,728 \\
\hline Baboon & 881,562 & $1,372,420$ & 490,858 & $1,083,371$ & $1,323,995$ & 240,624 & 556 & 753,170 & 752,614 \\
\hline Pepper & 18,766 & $1,184,195$ & $1,165,429$ & 187,503 & $1,067,848$ & 880,345 & 557 & 139,943 & 139,386 \\
\hline
\end{tabular}




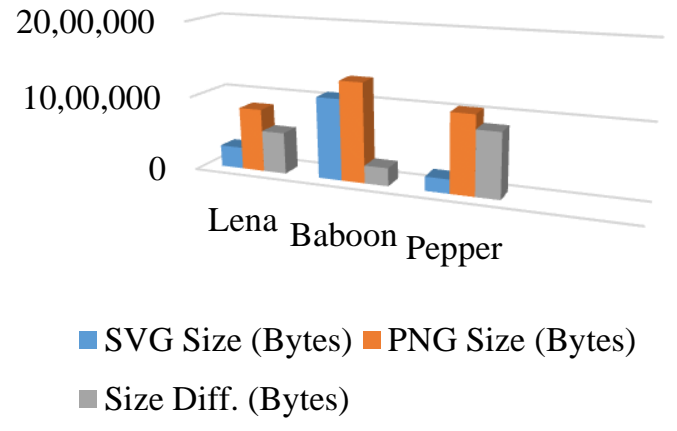

Fig. 4. SVG and PNG image sizes in Visio image editing software.

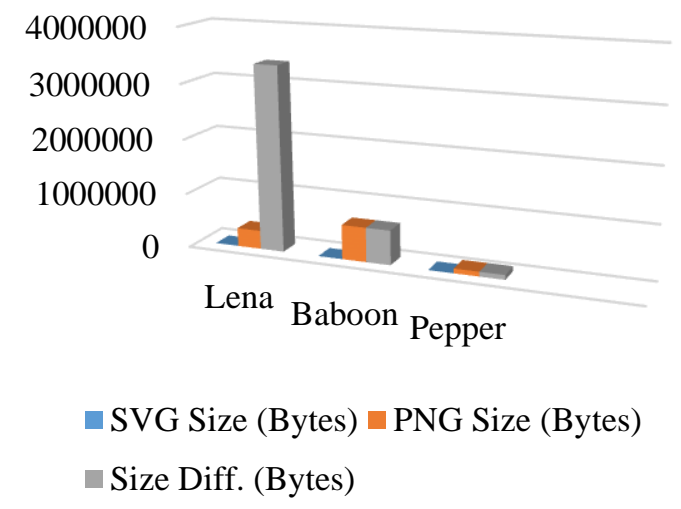

Fig. 5. SVG and PNG image sizes in Dia image editing software.

In addition, Table III above shows that SVG image format occupies less capacity than PNG image format after steganography processing, which evidences that SVG image format is more efficient than PNG image format as regards image's capacity. For example, the size of Lena image that is saved in SVG image format using Inkscape software after steganography processing is 562,628 Bytes, while its size is $1,825,337$ Bytes when saved in PNG image format. The difference in size of Lena image between SVG and PNG image formats is 1,262,709 Bytes, which acts $69.2 \%$ of PNG image's size. Similarly, the size of Lena image that is saved in SVG image format using Visio software after steganography processing is 288,681 Bytes, while its size is 845,107 Bytes when saved in PNG image format. The difference in size of Lena image between SVG and PNG image formats is thus, 556,426 Bytes, which is $65.8 \%$ of PNG image's size. In the same way, the size of Lena image that is saved in SVG image format using Dia software after steganography processing is 554 Bytes, while its size is 343,282 Bytes when it is saved in PNG image format. The difference in size of Lena image between SVG and PNG image formats is thus 342,728 Bytes, which is $99.6 \%$ of PNG image's size. Likewise, identical calculations are applied to all other images as shown in Table III above. Overall, the difference in size between SVG and PNG image formats after steganography processing is about $18.2 \%-99.9 \%$. Correspondingly, Fig. 6, 7, and 8 show the sizes of SVG and PNG image formats and the difference between them in Inkscape, Visio, and Dia image editing software respectively after steganography processing.

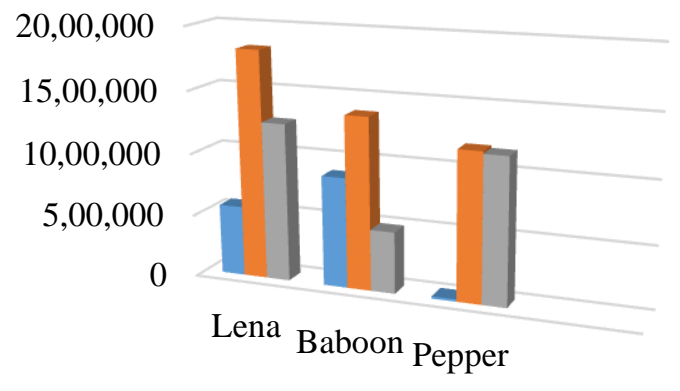

๑ SVG Size (Bytes) $\backsim$ PNG Size (Bytes)

$\square$ Size Diff. (Bytes)

Fig. 6. SVG and PNG image sizes in Inkscape image editing software after steganography processing.

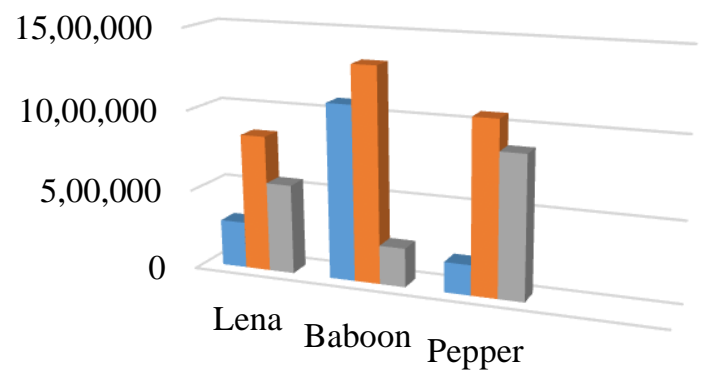

$\square$ SVG Size (Bytes) $₫$ PNG Size (Bytes)

$\square$ Size Diff. (Bytes)

Fig. 7. SVG and PNG image sizes in Visio image editing software after steganography processing.

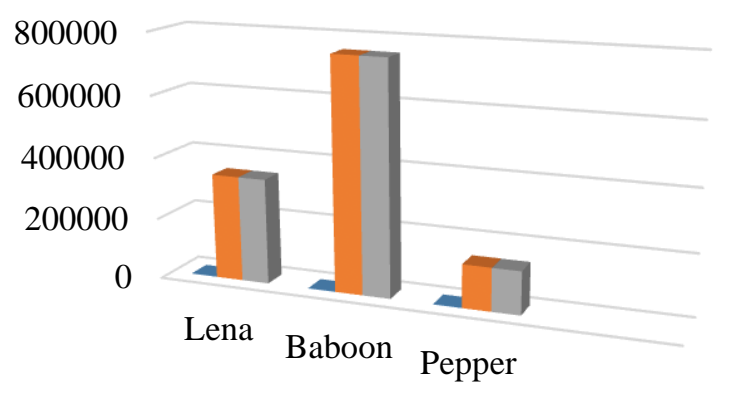

$\llbracket$ SVG Size (Bytes) $\square$ PNG Size (Bytes)

$\square$ Size Diff. (Bytes)

Fig. 8. SVG and PNG image sizes in Dia image editing software after steganography processing.

In addition, the following Tables IV, V, and VI evidence that SVG image format occupies less capacity in comparison to PNG image format after steganography processing. Thus, it can be inferred that SVG image format is more efficient than PNG image format in term of image's capacity. 
TABLE IV. SVG AND PNG IMAGES SIZE DiFFERENCE BEFORE AND AFTER STEGANOGRAPHY PROCESSING

\begin{tabular}{|c|c|c|c|c|c|}
\hline \multirow[t]{2}{*}{ Image Name } & \multirow[t]{2}{*}{ Before/After Steganography Processing } & \multicolumn{4}{|c|}{ Inkscape Image Editing Software } \\
\hline & & SVG Size (Bytes) & SVG Diff. (Bytes) & PNG Size (Bytes) & PNG Diff. (Bytes) \\
\hline \multirow{2}{*}{ Lena } & Before & 562,514 & \multirow{2}{*}{114} & $1,275,975$ & \multirow{2}{*}{549,362} \\
\hline & After & 562,628 & & $1,825,337$ & \\
\hline \multirow{2}{*}{ Baboon } & Before & 881,448 & \multirow{2}{*}{114} & $1,161,746$ & \multirow{2}{*}{210,674} \\
\hline & After & 881,562 & & $1,372,420$ & \\
\hline \multirow{2}{*}{ Pepper } & Before & 18,652 & \multirow{2}{*}{114} & 645,490 & \multirow{2}{*}{538,705} \\
\hline & After & 18,766 & & $1,184,195$ & \\
\hline
\end{tabular}

TABLE V. SVG AND PNG IMAGES SIZE DIFFERENCE BEFORE AND AFTER STEGANOGRAPHY PROCESSING

\begin{tabular}{|c|c|c|c|c|c|}
\hline \multirow{2}{*}{$\begin{array}{l}\text { Image } \\
\text { Name }\end{array}$} & \multirow{2}{*}{$\begin{array}{c}\text { Before/After } \\
\text { Steganography } \\
\text { Processing }\end{array}$} & \multicolumn{4}{|c|}{ Visio Image Editing Software } \\
\hline & & SVG Size (Bytes) & SVG Diff. (Bytes) & PNG Size (Bytes) & PNG Diff. (Bytes) \\
\hline \multirow{2}{*}{ Lena } & Before & 288,569 & \multirow{2}{*}{112} & 841,419 & \multirow{2}{*}{3,688} \\
\hline & After & 288,681 & & 845,107 & \\
\hline \multirow{2}{*}{ Baboon } & Before & $1,083,259$ & \multirow{2}{*}{112} & $1,322,223$ & \multirow{2}{*}{1,772} \\
\hline & After & $1,083,371$ & & $1,323,995$ & \\
\hline \multirow{2}{*}{ Pepper } & Before & 187,391 & \multirow{2}{*}{112} & $1,046,081$ & \multirow{2}{*}{21,767} \\
\hline & After & 187,503 & & $1,067,848$ & \\
\hline
\end{tabular}

TABLE VI. SVG AND PNG IMAGES SIZE DIFFERENCE BEFORE AND AFTER STEGANOGRAPHY PROCESSING

\begin{tabular}{|c|c|c|c|c|c|}
\hline \multirow{2}{*}{$\begin{array}{l}\text { Image } \\
\text { Name }\end{array}$} & \multirow{2}{*}{$\begin{array}{l}\text { Before/After } \\
\text { Steganography } \\
\text { Processing }\end{array}$} & \multicolumn{4}{|c|}{ Dia Image Editing Software } \\
\hline & & SVG Size (Bytes) & SVG Diff. (Bytes) & PNG Size (Bytes) & PNG Diff. (Bytes) \\
\hline \multirow{2}{*}{ Lena } & Before & 434 & \multirow{2}{*}{120} & 336,087 & \multirow{2}{*}{7,195} \\
\hline & After & 554 & & 343,282 & \\
\hline \multirow{2}{*}{ Baboon } & Before & 436 & \multirow{2}{*}{120} & 629,707 & \multirow{2}{*}{123,463} \\
\hline & After & 556 & & 753,170 & \\
\hline \multirow{2}{*}{ Pepper } & Before & 437 & \multirow{2}{*}{120} & 102,015 & \multirow{2}{*}{37,928} \\
\hline & After & 557 & & 139,943 & \\
\hline
\end{tabular}

Table IV present the sizes of images that are saved in SVG and PNG formats using Inkscape image editing software before and after steganography processing. Thus, hiding the secret data "My Visa Credit Card number is 4539962848122779, CCV number is 483, and Expiration Date is $12 / 17$ " into SVG images format yields a fixed growth of image's size, which is 114 Bytes for all SVG images format, while it yields a variable image's size growth for PNG images format. This merit is achieved due to saving of the secret data outside SVG images format, and hence it provides a scalability. However, the secret data is when saved inside PNG images format, shows an increase in their sizes.

In addition, Table $\mathrm{V}$ present the sizes of images that are saved in SVG and PNG formats using Visio image editing software before and after steganography processing. Thus, hiding our secret data "My Visa Credit Card number is 4539962848122779, CCV number is 483, and Expiration Date is 12/17" into SVG images format yields a fixed growth of image's size, which is 112 Bytes for all SVG images format, while it yields a variable image's size growth for PNG images format. This merit is achieved due to saving a secret data outside SVG images format, and hence it provides a scalability. However, when the secret data is saved inside PNG images format, it shows an increase in their sizes.

Finally, Table VI shows sizes of images that are saved in SVG and PNG formats using Dia image editing software before and after steganography processing. Thus, hiding our secret data "My Visa Credit Card number is 4539962848122779, CCV number is 483, and Expiration Date is $12 / 17$ " into SVG images format yields a fixed growth of image's size, which is 120 Bytes for all SVG images format, while it yields a variable image's size growth for PNG images format. This merit is achieved due to saving the secret data outside SVG images format, and hence it provides a 
scalability. However, when the secret data is saved inside PNG images format, it shows an increase in their sizes.

\section{CONCLUSION}

The current research has been conducted with the purpose to draw a comparative study on steganography digital images. It focuses on a nominated raster image, namely Portable Network Graphics (PNG), and a nominated vector image, namely Scalable Vector Graphics (SVG) Images Formats. The purpose of study has been to deduce optimal choices for image steganography, especially those that support web services. As shown above, SVG images format has been revealed in the study to be more efficient for image steganography than PNG images format in terms of capacity, scalability, simplicity, and performance. As demonstrated, SVG images format occupies fewer sizes before and after steganography processing, which makes it lighter and smoother for exchange through networks. As well, SVG images format provides scalability to a secret data, since it saves it externally rather than PNG images format, which saves it internally. Finally, processing steganography in SVG images format is simpler than PNG images format, since SVG images format are merely text files that provide the ease of processing using any direct text editor software, while being processed through RGB color model, and hence help to boost performance for processing steganography.

\section{REFERENCES}

[1] D. T. M. M.J.Thenmozhi, "A New Secure Image Steganography Using Lsb And Spiht Based Compression Method," Int. J. Eng. Res. Sci., 2016.

[2] N. K. M. A.- Anjali Tiwari, Seema Rani Yadav, "A Review on Comparison between Different Image Steganography Techniques," vol. 3, no. 8, pp. 355-358, 2014.

[3] R. B. A. \& O. M. A.-Q. Nagham Hamid, Abid Yahya, "Image Steganography Techniques : An Overview," Int. J. Comput. Sci. Secur., no. 6, pp. 168-187, 2012.

[4] A. Almutairi, "Optimal Specifications for a Secure Image Steganography Method," Int. J. Emerg. Trends Technol. Comput. Sci., vol. 6, no. 2, pp. 198-202, 2017.

[5] Sakshica and D. K. Gupta, "Various Raster and Vector Image File Formats," Int. J. Adv. Res. Comput. Commun. Eng., vol. 4, no. 3, pp. 268-271, 2015.

[6] P. M. Nishad and R. Manicka Chezian, "Various Colour Spaces and Colour Space Conversion Algorithms," J. Glob. Res. Comput. Sci., vol. 4, no. 1, pp. 44-48, 2013.

[7] H. K. Kelda and P. Kaur, "A Review: Color Models in Image Processing,” Int. J. Comput. Technol. Appl., vol. 5, no. 2, pp. 319-322, 2014.

[8] M. J. Dahan, N. Chen, A. Shamir, and D. Cohen-Or, "Combining color and depth for enhanced image segmentation and retargeting," Vis. Comput., vol. 28, no. 12, pp. 1181-1193, 2012.

[9] A. C. Ã, J. Condell, K. Curran, P. M. Kevitt, A. Cheddad, J. Condell, K Curran, and P. Mc Kevitt, "Digital image steganography: Survey and analysis of current methods," Signal Processing, vol. 90, no. 3, pp. 727$752,2010$.
[10] C. Seel-audom, W. Naiyapo, and V. Chouvatut, "A search for geometric-shape objects in a vector image: Scalable Vector Graphics (SVG) file format," in 2017 9th International Conference on Knowledge and Smart Technology (KST), 2017, pp. 305-310.

[11] R. M. Mathis, "Constraint scalable vector graphics, accessibility and the semantic Web," in Proceedings. IEEE SoutheastCon, 2005., 2005, pp. 588-593.

[12] D. M. Cabrita and W. Godoy, "PNG Optimization Techniques Applied to Lossless Web Images," IEEE Lat. Am. Trans., vol. 10, no. 1, pp. 1398-1401, Jan. 2012.

[13] H. Mao, Z. Hu, L. Zhu, and H. Qin, "PNG File Decoding Optimization Based Embedded System," in 2012 8th International Conference on Wireless Communications, Networking and Mobile Computing, 2012, pp. $1-4$.

[14] Y. Jiazheng, H. Jinghua, W. Yujian, and B. Hong, "Converting real images to SVG based on XML," in IET 2nd International Conference on Wireless, Mobile and Multimedia Networks (ICWMMN 2008), 2008, pp. 360-363.

[15] Z. Liu, X. Cheng, C. Jia, and J. Yang, "Format Compliant Degradation for PNG Image," in 2013 Fourth International Conference on Emerging Intelligent Data and Web Technologies, 2013, pp. 720-723.

[16] A. Jain and V. Jain, "PNG image copyright protection and authentication using SVD hash and AES," in 2014 International Conference on Advances in Engineering Technology Research (ICAETR - 2014), 2014, pp. 1-6.

[17] T. Kumar and K. Verma, "A Theory Based on Conversion of RGB image to Gray image," Int. J. Comput. Appl., vol. 7, no. 2, p. 9758887, 2010.

[18] J. S. Kang, Y. You, M. Y. Sung, T. T. Jeong, and J. Park, "Mobile Mapping Service Using Scalable Vector Graphics on the Human Geographic," in Seventh IEEE/ACIS International Conference on Computer and Information Science (icis 2008), 2008, pp. 672-677.

[19] C. Concolato, J. Le Feuvre, and J. C. Moissinac, "Design of an efficient scalable vector graphics player for constrained devices," IEEE Trans. Consum. Electron., vol. 54, no. 2, pp. 895-903, May 2008.

[20] I. Team, "Inkscape." 2017.

[21] Microsoft, "Visio Professional 2016." 2017.

[22] D. D. E. Team, "Dia Diagram Editor." 2017.

[23] S. Vaidya, "OpenStego." 2017.

[24] S. Singh and V. K. Attri, "Dual Layer Security of data using LSB Image Steganography Method and AES Encryption Algorithm," Int. J. Signal Process. Image Process. Pattern Recognit., vol. 8, no. 5, p. 259266, 2015.

[25] M. R. Shende; and A. Welekar, "Advanced Steganography for Hiding Data and Image using AudioVideo," Int. J. Recent Innov. Trends Comput. Commun., 2016.

[26] Sahar A. El_Rahman, "A Comprehensive Image Steganography Tool using LSB Scheme," I.J. Image, Graph. Signal Process., 2015.

[27] D. Rawat and V. Bhandari, "Steganography Technique for Hiding Text Information in Color Image using Improved LSB Method," Int. J. Comput. Appl., vol. 67, no. 1, p. 9758887, 2013.

[28] Raju and Mohit Dhanda, "An Improved LSB based Image Steganography for Grayscale and Color Images," Int. J. Curr. Eng. Technol., vol. 5, no. 5, p. 22774106, 2015.

[29] Mohit, "An Enhanced Least Significant Bit Steganography Technique," Int. J. Adv. Res. Comput. Eng. Technol., vol. 5, no. 6, p. 22781323, 2016.

[30] K. Muhammad;, J.Ahmad;, N. U. Rehman; Z. Jan;, and R. J. Qureshi, "A Secure Cyclic Steganographic Technique for Color Images using Randomization,” Tech. Journal, Univ. Eng. Technol. Taxila, 2014. 